\title{
PERCEPCIÓN DE LA INCLUSIÓN EDUCATIVA EN EL CONTEXTO UNIVERSITARIO: UN ESTUDIO APROXIMATIVO EN LA FCEE DE GRANADA
}

\section{PERCEPTION OF THE EDUCATIONAL INCLUSION IN THE UNIVERSITY CONTEXT: AN APPROXIMATE STUDY IN THE FCEE OF GRANADA}

Conde Lacárcel, A. (Universidad de Granada)

ISSN : 2254-5883

\author{
Revista electrónica de investigación Docencia Creativa \\ Fecha de recepción: 28 de mayo de 2012 \\ Fecha de revisión: 1 de junio de 2012 \\ Fecha de aceptación: 11 de junio de 2012
}

\begin{abstract}
Resumen
El presente artículo pretende realizar un análisis aproximativo respecto al grado de aplicación de la inclusión educativa en la Facultad de Ciencias de la Educación de la ciudad de Granada. ¿Es consciente el alumnado y el profesorado de esta corriente que se está desarrollando desde hace tiempo en el sistema educativo de otros países y también en el nuestro; En qué grado conciben la comunidad universitaria que está llevándose a cabo? Primera parte, de un estudio aproximativo centrado en la percepción del alumnado, que posteriormente se complementará con la percepción del profesorado.
\end{abstract}

Palabras clave: educación universal, acceso a la educación, inclusión educativa, enseñanza superior.

\section{Abstract}

The present article tries to realize an approximate analysis with regard to the grade of application of the educational inclusion in the Faculty of Sciences of the Education of the city of Granada. There are conscious the pupils and professorship of this current that is developing for time in the educational system of other countries and also in ours; in what grade do they conceive the university community that is carried out? The first part, of the approximate study centered on the perception of the university pupils, which later will complement itself with the perception of the professorship.

Keywords: universal education, access to education, inclusive education, higher education.

\section{LA EDUCACIÓN INCLUSIVA}

La educación para todos en igualdad de condiciones, de calidad, superadora de las injusticias sociales y modificadora de nuestro entorno como herramienta emancipadora para las personas, ha sido una de las ideas que han hecho evolucionar la conceptualización de la educación en general, y la Educación Especial en particular desde perspectivas categorizadoras y remediales hacia otras formas de acción integradoras. 


\section{ReiDoCrea. Volumen 1}

Ahora se habla de Inclusión educativa en nuestros centros y aulas. Según Stainback y Stainback (1990), la escuela inclusiva se puede definir como: "un lugar en el que todo el mundo se siente aceptado, apoyado y ayudado por sus compañeros, profesores y otros miembros de la comunidad escolar y donde se da respuesta a sus necesidades educativas".

La historia de este movimiento, sienta sus bases en las declaraciones e informes promulgados por organismos internacionales; la evolución en la intervención de las personas con necesidades educativas especiales; la iniciativa de educación regular estadounidense (Regular Education Iniciative); el Informe Warnok y de una manera más significativa en la Declaración de Salamanca de 1994 sobre Necesidades Educativas Especiales.

Desde estas iniciativas, se concibe la educación inclusiva como un intento de transformar el sistema educativo para que todos los niños/as, jóvenes y adultos se aseguren el máximo desarrollo, independientemente de sus características particulares (con o sin discapacidad), sociales, económicas, religiosas, etc., de manera que se superen las desigualdades educativas.

Para conseguirlo y siguiendo las ideas de Booth, T. (2000) en su obra Índex for Inclusión: es preciso modificar la cultura, las políticas y prácticas educativas de los centros y comunidades educativas, institutos, universidades, etc. estableciendo y organizando todos los recursos personales, materiales, institucionales...necesarios en los contextos comunitarios en que viven, crecen y aprenden.

Esta iniciativa, ha ido extendiéndose con mayor fuerza desde el año 2000 con la aparición de esta obra en Reino Unido a países europeos como Noruega, Alemania o Finlandia, entre otros, además de trabajarse en adaptaciones a contextos tan dispares como pueden ser Australia, Brasil, Sudáfrica o la India; por su parte, América Latina y el Caribe, a través de la Oficina Regional de la Unesco está liderando las experiencias en este sentido; incluyendo el contexto universitario, de acuerdo con la información que podemos encontrar en el Prólogo de la Guía para la evaluación y mejora de la educación inclusiva realizada por el Consorcio Universitario para Educación Inclusiva aquí en España y otros documentos que mencionaremos posteriormente.

No se trata de diagnosticar, categorizar e implementar las soluciones de forma individual como se venía haciendo hasta ahora en un enfoque tradicional, sino de actuar en el contexto de manera que se superen todas las barreras al aprendizaje y se responda a las necesidades de todos.

De acuerdo con la UNESCO (2000): "La educación inclusiva se basa en el derecho de todos los alumnos a una educación de calidad que responda a las necesidades básicas de aprendizaje y enriquezca sus vidas. Centrándose en particular en grupos vulnerables y marginados."

En este tipo de escuela el alumnado con NEE se incluye en las aulas ordinarias para favorecer una vida lo más normalizada posible, incorporando las ayudas que sean precisas tanto para alumnos/as con alguna discapacidad como para alumnos/as "normales". Contempla, según López Villegas, María, (2010) citando a Giangreco, Cloninger y col. (1994): 
- Unos agrupamientos heterogéneos con una proporción natural de alumnos con discapacidad y sin ella en el aula, centro ordinario, distintos niveles de desarrollo que son educados juntos.

- Un sentido de pertenencia al grupo.

- Una instrucción de nivel múltiple.

- Un uso de ambientes frecuentados por personas sin discapacidad.

- Experiencias educativas equilibradas.

En nuestro país, España, será a partir de la Ley Orgánica de Educación (LOE) cuando empiece a realizar su andadura la Inclusión educativa con encomiables esfuerzos, aunque todavía con numerosas barreras, desconocimientos y experiencias muy localizadas, como la de Salas y Domínguez (1999) o Cereza (2000) centradas fundamentalmente en los niveles de primaria, secundaria y educación de adultos.

De todas formas podemos encontrar revisiones teóricas como la de Jurado y Sánchez, (2000) que cuestionan el diseño curricular tradicional o las diversificaciones curriculares en secundaria; y materiales específicos y estrategias didácticas inclusivas como por ejemplo, la de la sección de documentos sobre inclusión educativa de la página web de la Federación Vasca de Asociaciones en favor de las personas con discapacidad intelectual (http://www.fevas.org) o la labor desarrollada en forma de Proyectos de colaboración con Iberoamérica del M.E.C. por medio de "un modelo de cooperación [...] apoyo y acompañamiento institucional entre ministerios de educación, [...] relación intersectorial y de la sociedad civil, la capacitación y formación de docentes, los seminarios y encuentros". (Extraído el 31-1-2012 de: http://www.educacion.gob.es/educacion/actividadinternacional/cooperacion-educativa/proyec-educa-inclus-iberoamerica.html)

En el contexto universitario, las temáticas tanto a nivel teórico como de experiencias, más desarrolladas y relacionadas con la inclusión atienden principalmente a:

- el liderazgo educativo.

- la calidad educativa.

- la convivencia y resolución de conflictos.

- la exclusión por razón de género.

- la interculturalidad.

- las comunidades de aprendizaje.

- la formación del profesorado.

- la cultura de colaboración.

Centrándonos en este ámbito por la necesidad de concreción a pesar de la cantidad de información de la que disponemos, la inclusión educativa en contextos universitarios 
parece ser un proceso fundamentalmente desarrollado en los países Iberoamericanos y del Caribe, con experiencias como por ejemplo la que podemos recoger del 1er Congreso Latino-americano de Ciencias de la Educación: "Actitudes de Docentes Universitarios hacia los Procesos de Inclusión Educativa de Alumnos con Capacidades diferentes en la Universidad Autónoma de San Luís Potosí" de Vázquez Martínez, B. , Méndez Pineda, J. y Mendoza Saucedo, F. (2010); o también en Canadá con el Proyecto "Conexiones" para favorecer la inclusión de alumnado con necesidades especiales en escuelas y centros universitarios y la formación de docentes sobre inclusión en las Universidades de Moncton y Prince Edward Island.

Pero, ¿qué podemos encontrar a este respecto en España? Desde el CERMI (Comité Español de Representantes de Personas con Discapacidad), se ha generado una iniciativa muy interesante: desarrollar un observatorio estatal sobre inclusión, en el que se elaborará un informe bianual para comprobar la realidad de su desarrollo en el contexto universitario.

Teniendo en cuenta todo, se ha pensado hacer una encuesta aproximativa, con el fin de establecer una panorámica de la realidad que podemos encontrarnos en la Facultad de Ciencias de la Educación de la Universidad de Granada.

¿Hasta qué punto los futuros docentes están formándose a este respecto; qué ideas tienen respecto a la inclusión educativa; cómo la transmiten los profesores universitarios a sus alumnos y alumnas en las distintas prácticas educativas llevadas a cabo a nivel teórico, en sus seminarios, etc. de acuerdo con el Espacio Europeo de Educación Superior?

\section{MÉTODO Y MATERIALES}

Para responder a las preguntas planteadas y dar forma a un primer escenario con el que trabajar la inclusión educativa a nivel universitario en nuestra facultad, hemos llevado a cabo una metodología por encuesta vía on-line, sobre las distintas opiniones y valoraciones que tiene la muestra seleccionada.

En la elaboración del instrumento utilizado, hemos llevado a cabo una selección de ítems del "Cuestionario del Índice de Inclusión" del Programa de Educación Inclusiva con Calidad llevado a cabo por el Ministerio de Educación Nacional de Colombia, debido a su validez y fiabilidad, sin necesidad de llevar a cabo modificaciones en la formulación de los distintos ítems.

Utilizamos una escala Lickert con una graduación de respuesta de 1 a 5 (1. No sé. 2. No se hace. 3. Algunas veces. 4. Casi siempre. 5. Siempre), con un porcentaje de fiabilidad del $95 \%$. La difusión de la misma y el tratamiento de los datos han sido facilitados por la plataforma encuestafacil.com que nos permite descargarlos en fichero csv y los formatos Excel y Access los datos cuantitativos y trabajarlos con el programa estadístico SPSS (en este caso v.17.0).

El Universo teórico objeto del estudio está formada por el conjunto de la comunidad universitaria de la Facultad de Ciencias de la Educación (en este caso alumnado), siendo seleccionada de manera aleatoria para una mayor validez de los datos recogidos y evitar 


\section{ReiDoCrea. Volumen 1}

falseamientos de la información o posibles errores. Al tener un carácter voluntario, la muestra final ha sido la siguiente:

- Cuestionarios entregados vía-online: 104.

- Cuestionarios contestados: 72.

El cuestionario original sobre el que hemos realizado la adaptación, nos proporciona para un nivel de fiabilidad del 95\% la necesidad de una población mínima de entre 61 a 100 individuos y una muestra de 55 individuos para obtener un índice real de los datos recogidos. Al tratarse de un estudio aproximativo, con el fin de realizar una primera revisión del escenario para una posible investigación posterior, podemos considerar que los datos iniciales pueden proporcionarnos "pistas mínimamente fiables" sobre el grado de conocimiento en los estudiantes sobre el estado de la inclusión en su facultad de educación.

Los ítems seleccionados hacen referencia a las áreas de gestión directiva $(A)$, gestión académica (B) y de gestión de la comunidad (D):

- Direccionamiento estratégico y horizonte institucional: Misión, visión y principios institucionales (A.1.1) y metas institucionales (A.1.2).

- Gerencia estratégica: estrategia pedagógica (A.2.3); uso de información interna y externa para la toma de decisiones (A.2.4) y seguimiento y evaluación (A.2.5).

- Clima escolar: manejo de conflictos y casos difíciles (A.5.8).

- Gestión de aula: estilo pedagógico (B.3.3).

- Proyección a la comunidad: escuela de padres/madres (D.2.1); oferta de servicios a la comunidad (D.2.2) y servicio social estudiantil (D.2.3). 


\section{RESULTADOS}

En el área de GESTIÓN DIRECTIVA: Los resultados obtenidos, parecen indicar una percepción positiva de los estudiantes sobre el grado de inclusión de las personas a la hora de admitir a toda la población sin discriminación de ningún tipo, un 69’44\% eligieron la opción "Siempre" o "Casi siempre"; siendo ligeramente menos favorable a la hora de facilitar una adecuada atención a sus necesidades de aprendizaje, participación y convivencia el 68'06\% eligieron "Algunas veces" o "Casi siempre".

Resulta paradójico sin embargo, que cuando se les pregunta sobre el grado de conocimiento de los resultados del índice de inclusión a todos los agentes y grupos implicados un 70'83\% respondieron que "No lo sabían" o "No se hace".

\begin{tabular}{|c|c|c|}
\hline \multirow{2}{*}{$\begin{array}{l}\text { 1. La institución educativa admite a toda la población del sector sin } \\
\text { discriminación de raza, cultura, género, ideologia, credo, preferencia } \\
\text { sexual, condición socioeconómica, o situaciones de vulnerabilidad } \\
\text { como, necesidades educativas especiales por disc }\end{array}$} & \multicolumn{2}{|l|}{ Análisis técnico } \\
\hline & Media & 4,000 \\
\hline \multirow{7}{*}{ A.1.1 } & $\begin{array}{l}\text { Intervalo de confianza } \\
(95 \%)\end{array}$ & {$[3,767-4,233]$} \\
\hline & Tamaño de la muestra & 72 \\
\hline & Desviación típica & 1,007 \\
\hline & Error estándar & 0,119 \\
\hline & \multicolumn{2}{|c|}{ Conclusiones destacadas } \\
\hline & \multicolumn{2}{|l|}{$\begin{array}{l}\text { El "69,44\%" eligieron: } \\
\text { 5. Siempre. } \\
\text { 4. Casi siempre. }\end{array}$} \\
\hline & \multicolumn{2}{|c|}{$\begin{array}{l}\text { La opción menos elegida representa el } \\
\text { "2,78\%": } 1 \text {. No sé. }\end{array}$} \\
\hline
\end{tabular}


ReiDoCrea. Volumen 1

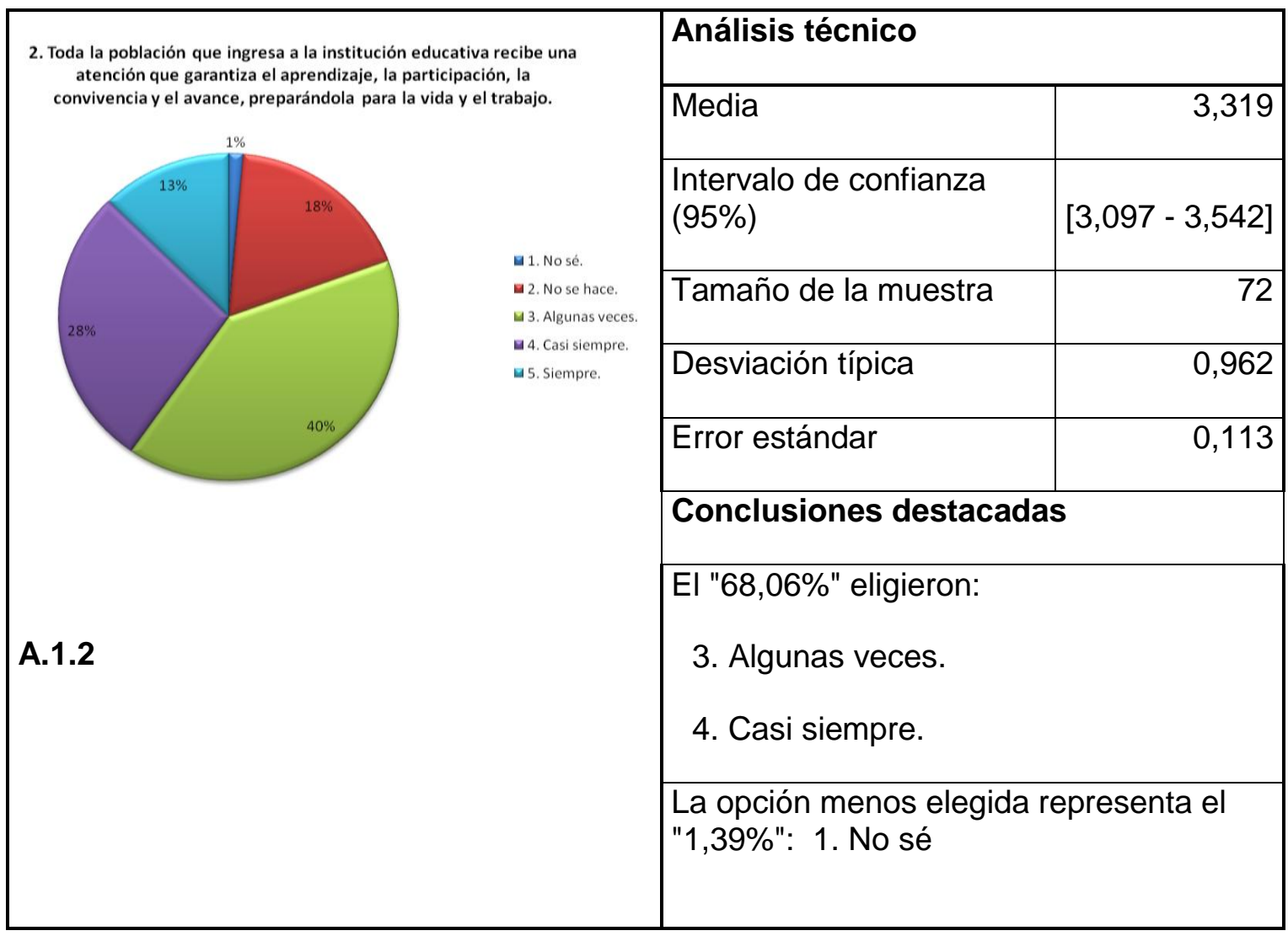

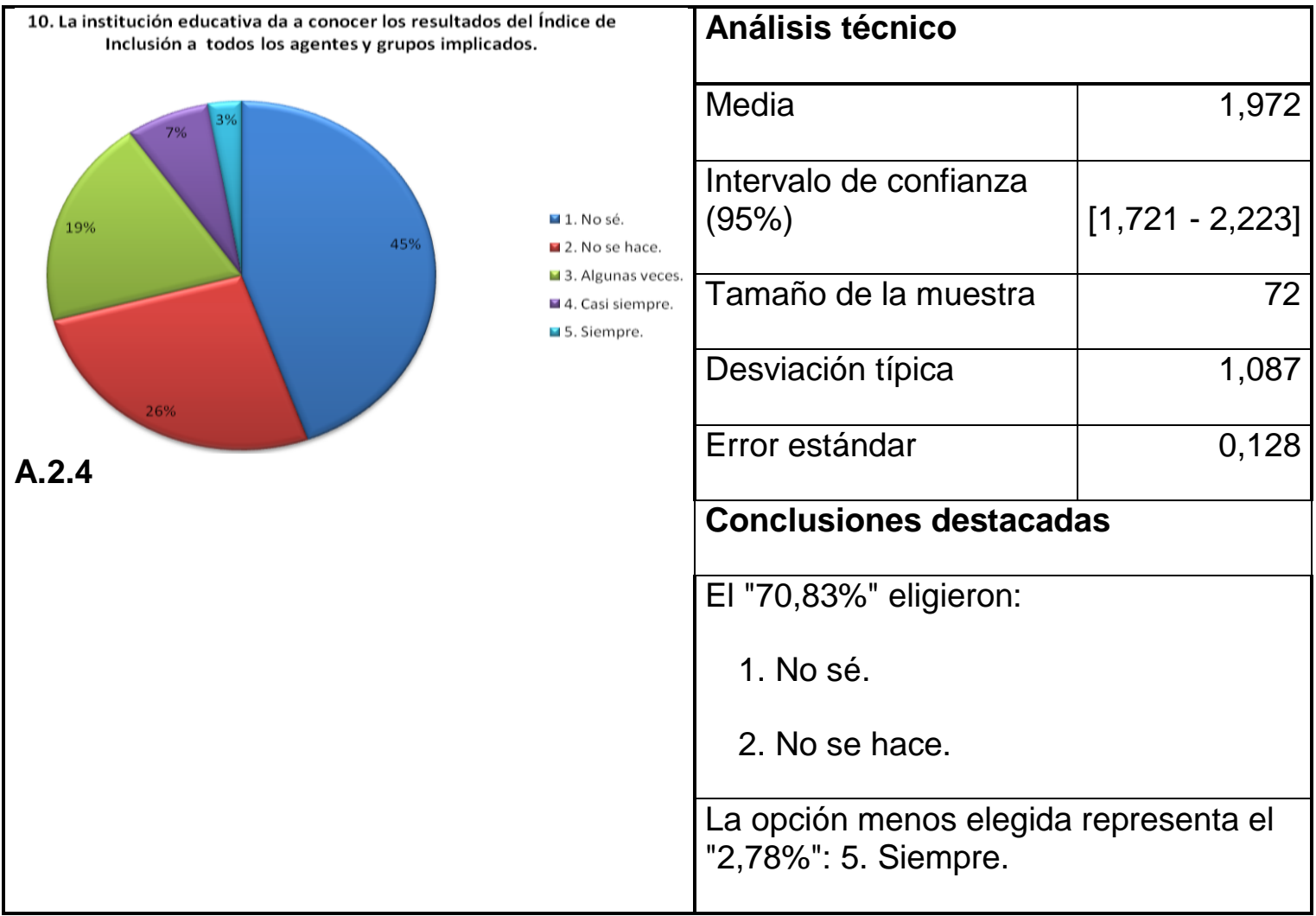


En las áreas de GERENCIA ESTRATÉGICA Y CLIMA ESCOLAR: En estas dos áreas, la percepción de los estudiantes es bastante pobre. A la pregunta sobre la satisfacción de las necesidades de aprendizaje, un 69'44\% indicaron que se realizaba "Algunas veces" o "Casi siempre". Un 65'28\% no sabían si se revisaban o se realizaban periódicamente las acciones encaminadas a la inclusión. El 79'17\% de los encuestados señalaron que sólo "Algunas veces" se les ayudaba a solucionar un posible problema o a mejorar sus aprendizajes.

¿Es esta la visión real que podemos encontrar en la FCEE de Granada? ¿Coincidirá con la visión de los profesores de la institución?

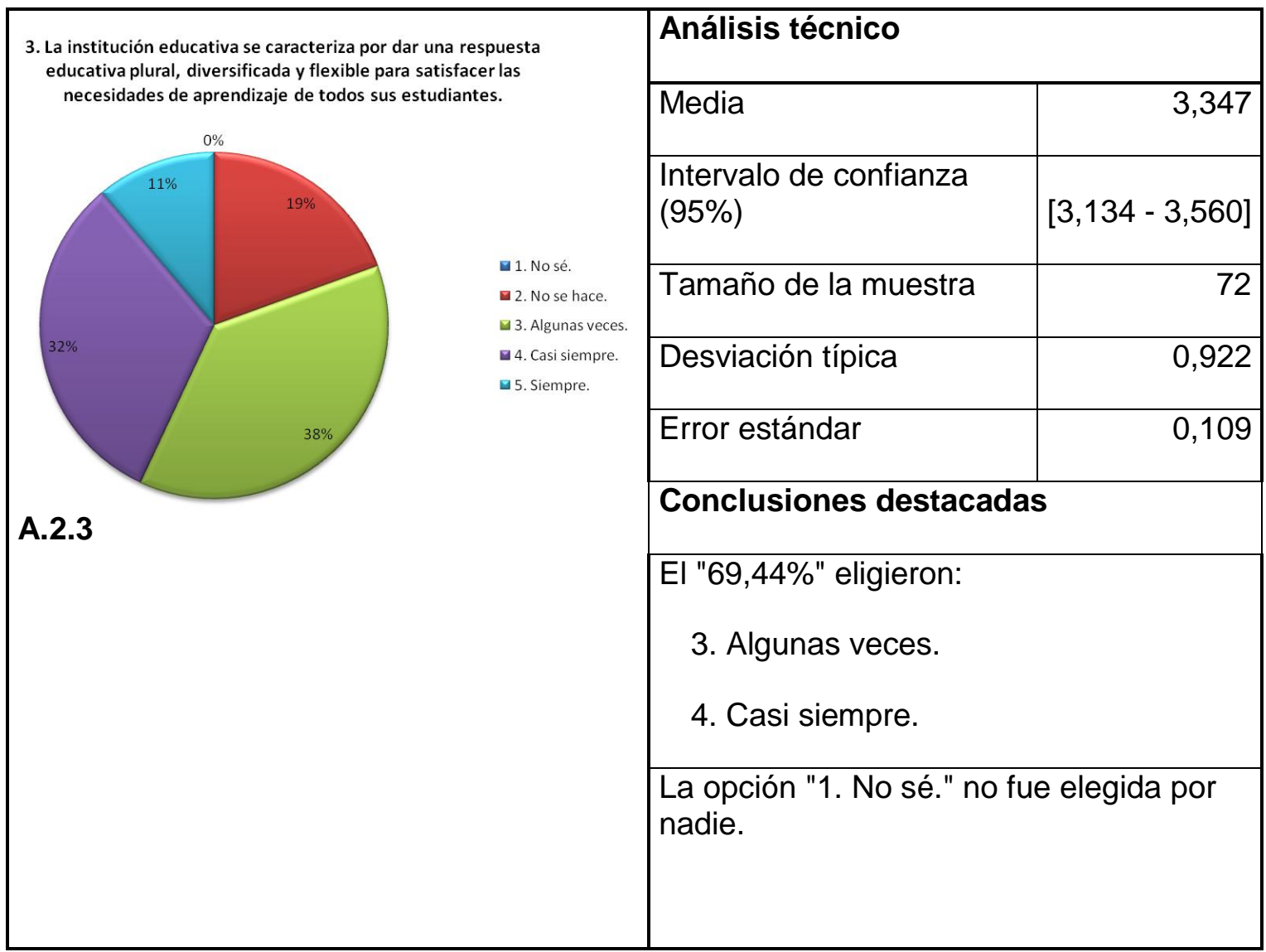


ReiDoCrea. Volumen 1

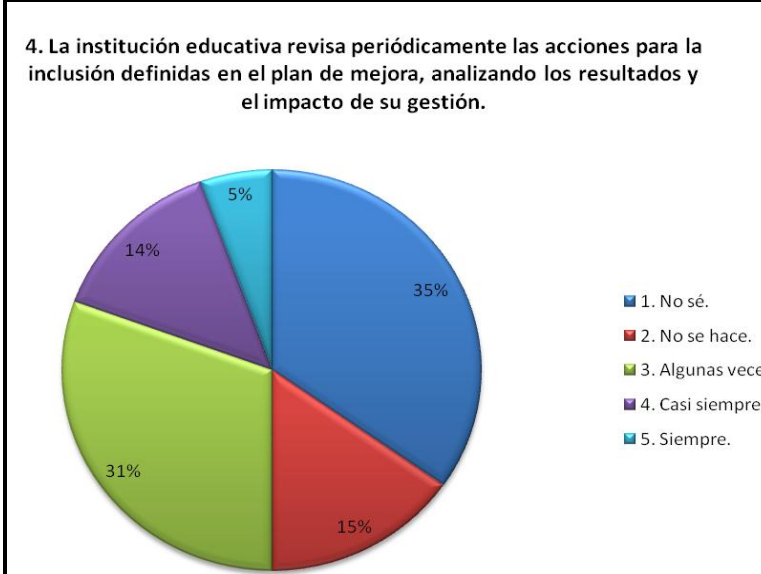

A.2.5

5. En la institución educativa cuando los estudiantes tienen problemas se les escucha, se les ayuda a solucionarlos y se les enseña a mejorar.

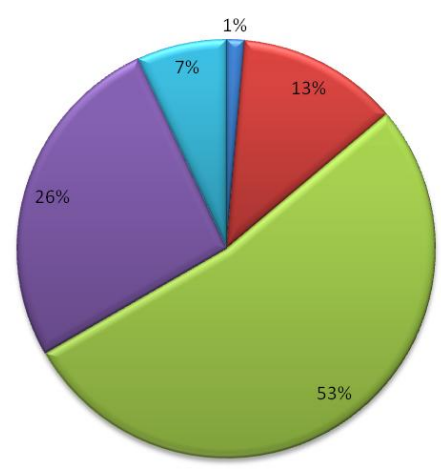

A.5.8
1 Nosé.

- 2. No se hace.

13. Algunas veces.

口. Casi siempre.

घ. Siempre.

\begin{tabular}{|l|r|}
\hline \multicolumn{2}{|l|}{ Análisis técnico } \\
\hline Media & 2,403 \\
\hline $\begin{array}{l}\text { Intervalo de confianza } \\
(95 \%)\end{array}$ & {$[2,114-2,692]$} \\
\hline Tamaño de la muestra & 1,252 \\
\hline Desviación típica & 0,148 \\
\hline Error estándar & \\
\hline
\end{tabular}

\section{Conclusiones destacadas}

El "65,28\%" eligieron:

1. No sé.

3. Algunas veces.

La opción menos elegida representa el "5,56\%":
Análisis técnico

\begin{tabular}{|l|r|}
\hline Media & 3,250 \\
\hline $\begin{array}{l}\text { Intervalo de confianza } \\
(95 \%)\end{array}$ & {$[3,061-3,439]$} \\
\hline Tamaño de la muestra & 0,818 \\
\hline Desviación típica & 0,096 \\
\hline Error estándar & \\
\hline
\end{tabular}

\section{Conclusiones destacadas}

El "79,17\%" eligieron:

3. Algunas veces.

4. Casi siempre.

La opción menos elegida representa el "1,39\%": 
En el área de GESTIÓN DE AULA: Los resultados aquí tampoco son muy positivos, ya que el $766^{\prime} 39 \%$ del estudiantado cree que no se involucra lo suficiente a la hora de participar en la elección del temario, las actividades de clase o las opciones de evaluación.

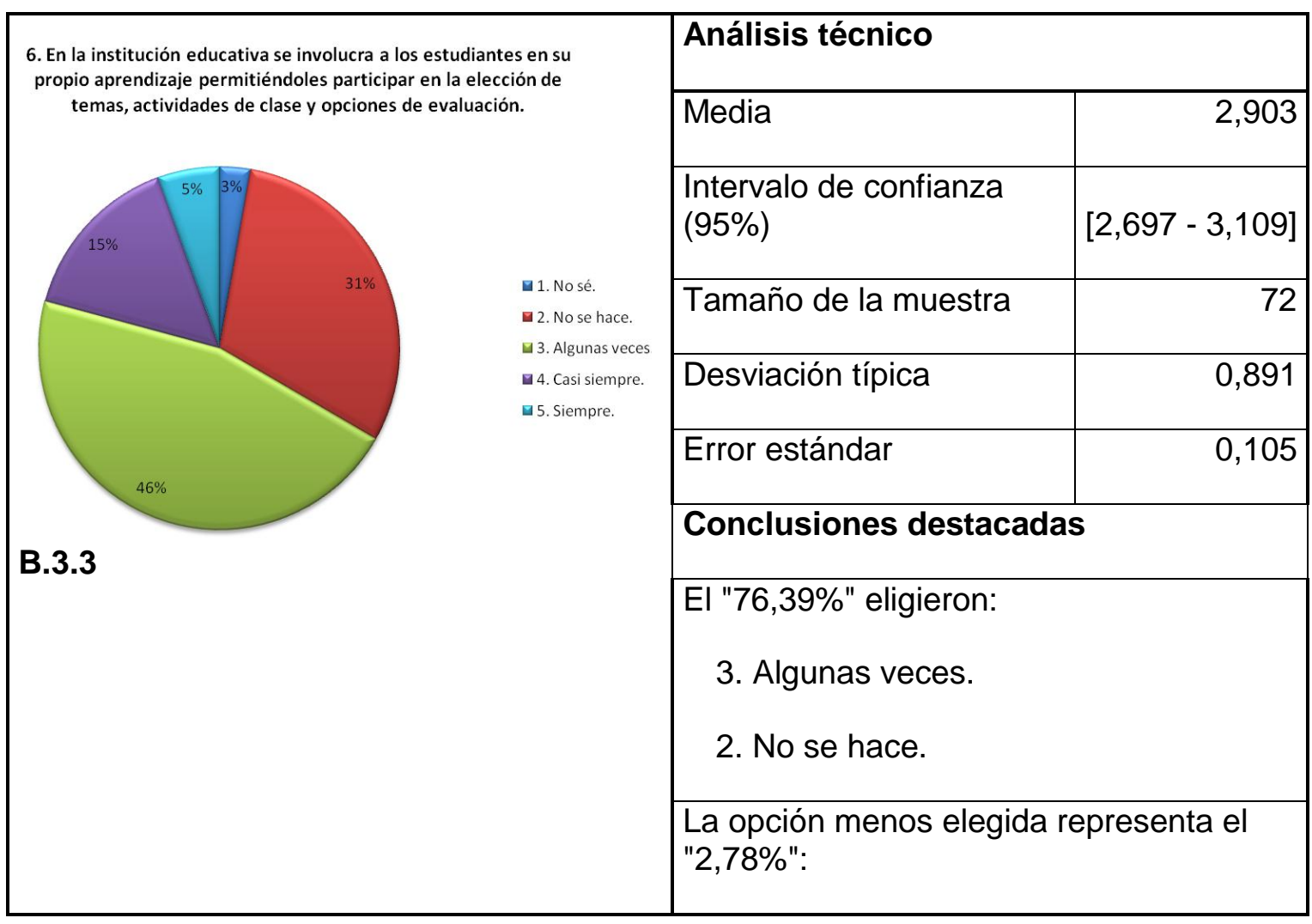


En el área de PROYECCIÓN A LA COMUNIDAD: No parece haber datos concluyentes ya que, un $80^{\prime} 56 \%$ de los estudiantes reparten sus respuestas a la pregunta sobre el desarrollo de temas y actividades inclusivas en su facultad con unos contradictorios "Algunas veces" o "Casi siempre", dos polos opuestos; se realizan o no se realizan pero no es posible decir que se realizan de manera esporádica al mismo tiempo que habitualmente ¿Tiene que ver esta respuesta con el grado de implicación del profesorado respecto a la inclusión; qué unos docentes lo profundicen más que otros en sus aulas?

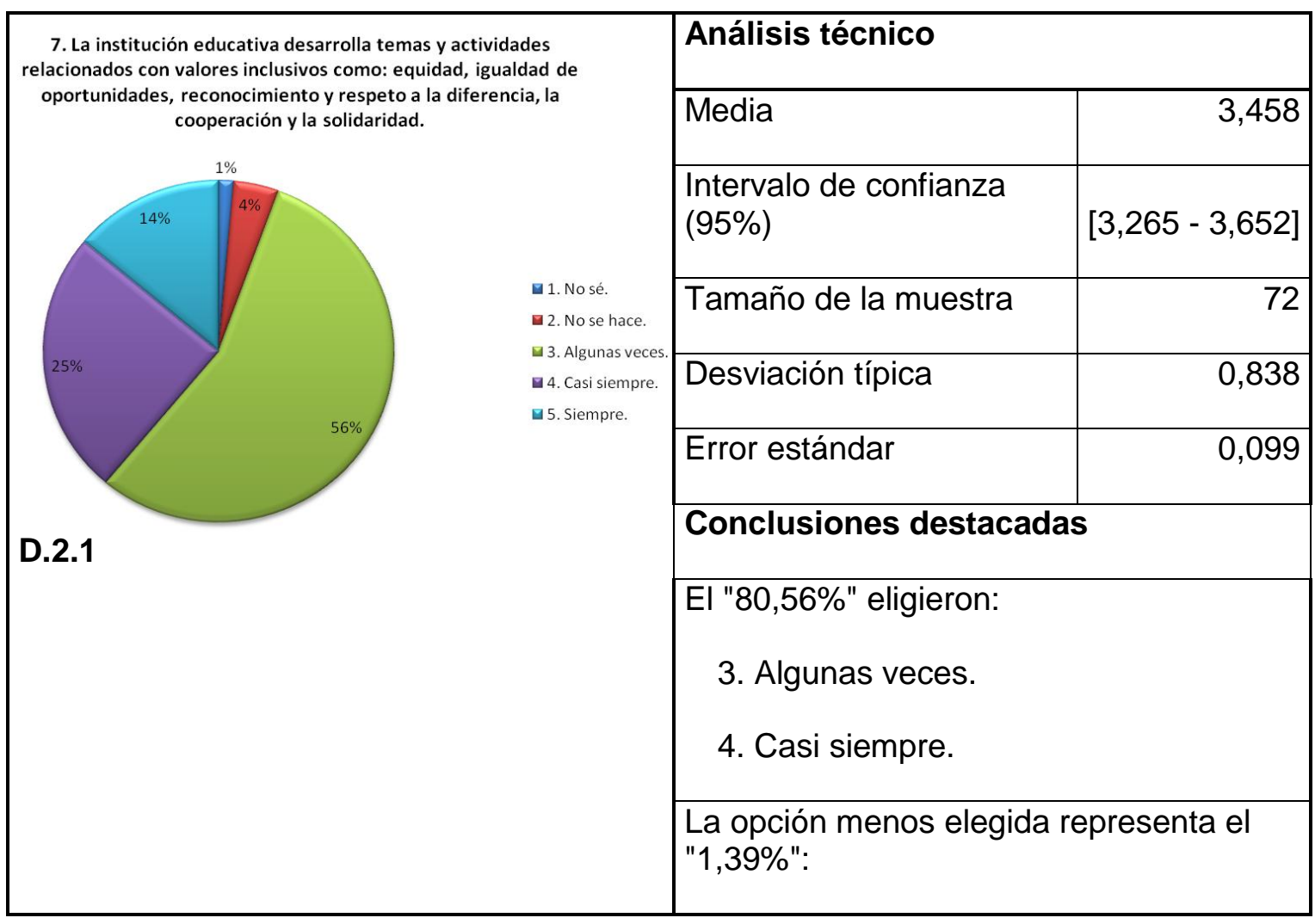

Igualmente ambiguo es el porcentaje de un 66 '67\% al que respondieron "Algunas veces" - "Casi siempre", cuando les preguntamos si se realizan actividades culturales, recreativas, deportivas y académicas con personas del sector para promover la inclusión,

Por último, el 70'83\% indicaron que no sabían si se promovían propuestas de acompañamiento entre estudiantes para ayudar a compañeros/as con diferentes situaciones que pudiesen favorecer la exclusión. 
ReiDoCrea. Volumen 1

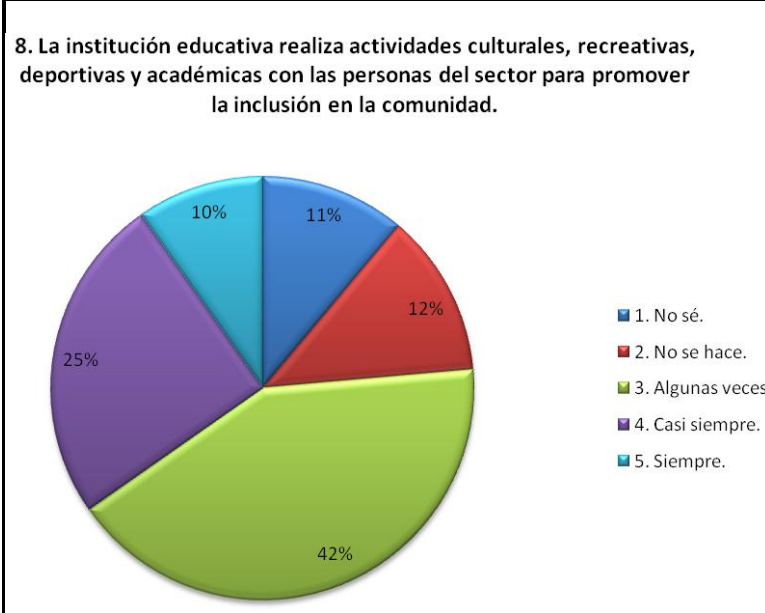

D.2.2

\begin{tabular}{|c|c|}
\hline \multicolumn{2}{|l|}{ Análisis técnico } \\
\hline Media & 3,097 \\
\hline $\begin{array}{l}\text { Intervalo de confianza } \\
(95 \%)\end{array}$ & {$[2,843-3,352]$} \\
\hline Tamaño de la muestra & 72 \\
\hline Desviación típica & 1,103 \\
\hline Error estándar & 0,130 \\
\hline \multicolumn{2}{|c|}{ Conclusiones destacadas } \\
\hline \multicolumn{2}{|l|}{ El "66,67\%" eligieron: } \\
\hline \multicolumn{2}{|l|}{ 3. Algunas veces. } \\
\hline \multicolumn{2}{|l|}{ 4. Casi siempre. } \\
\hline $\begin{array}{l}\text { La opción menos elegi } \\
\text { "9,72\%": }\end{array}$ & epresenta el \\
\hline
\end{tabular}

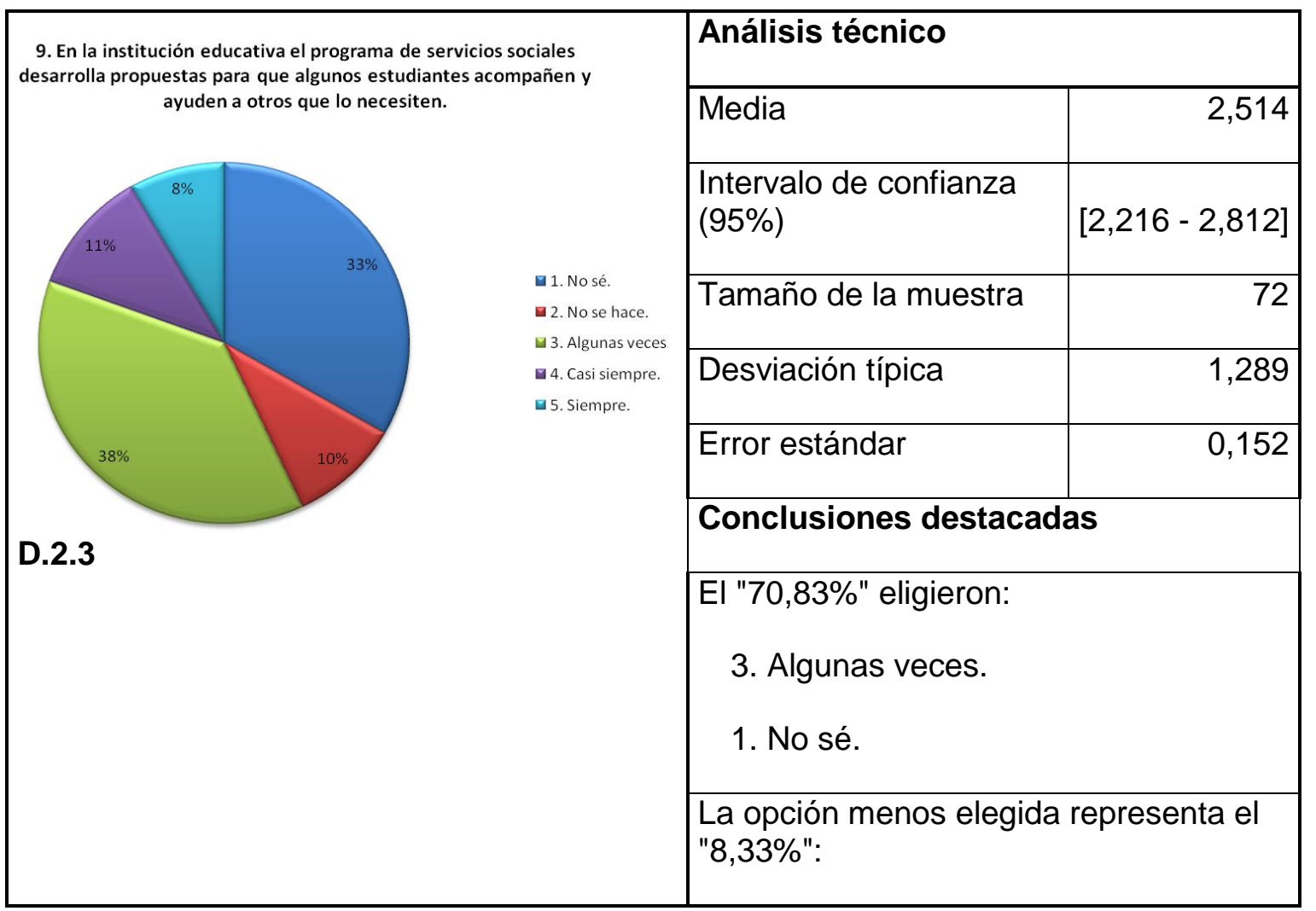




\section{DISCUSIÓN}

Aparentemente no parece haber una percepción clara sobre la inclusión por parte de los estudiantes.

A la hora de admitir alumnos/as en el centro, la percepción es que cualquiera puede acceder a los estudios universitarios en nuestra facultad siempre que se cumplan los distintos requisitos; aunque baja ligeramente cuando se habla de una adecuada atención a sus necesidades específicas, a la par que se desconocen los posibles índices de inclusión por parte de los grupos implicados o si hay propuestas de acompañamiento entre compañeros.

De manera generalizada, se cree que las actividades pensadas para favorecer las necesidades de aprendizaje, o resolver alguna cuestión problemática se llevan a cabo de una manera esporádica e irregular.

La necesidad de una mayor participación del alumnado en la elección de los temarios, las actividades de clase o las opciones de evaluación se hace palpable y la proyección a la comunidad no se percibe con claridad. El desarrollo de temáticas y actividades inclusivas de todo tipo (culturales, académicas, recreativas...) dentro de la facultad y con personas del sector, según el grupo de alumnos/as encuestados son minoritarias, según el estudiantado.

Teniendo en cuenta todos estos datos, podemos decir que se hace necesario realizar un análisis en profundidad por parte del conjunto de la comunidad educativa.

Seguidamente, la intención es llevar a cabo está aproximación en el profesorado y resto de cargos directivos y administrativos para contrastar las distintas percepciones y hacer una panorámica final. De todas formas, y a raíz de lo visto, recomiendo llevar a cabo un proceso completo de autoevaluación inclusiva con los materiales específicos existentes.

\section{REFERENCIAS BIBLIOGRÁFÍCAS:}

Booth, T., Ainscow, M., Black-Hawkins, K., Vaughan, M., \& Shaw, L. (2000). Índex for inclusion. Traducción castellana Guía para la evaluación y mejora de la educación inclusiva. Madrid. Consorcio Universitario para la Educación Inclusiva (2002)

La educación inclusiva como un modelo para la Educación para Todos (s.f.) En Como Modelo para la Educación para Todos de Inclusióneducativa.org. (Extraído el 30-1-2012 de http://www.inclusioneducativa.org/ise.php?id=5)

López Villegas, María (2010). Necesidades educativas especiales e Inclusividad. En Necesidades Específicas de Apoyo Educativo No Asociadas a Discapacidad. Infantil, Primaria y Secundaria. Editorial Universitas S.A. Madrid.

Ministerio de Educación Nacional. (2008). Índice de inclusión. Programa de Educación Inclusiva con calidad "Construyendo capacidad institucional para la atención a la Diversidad". Revolución Educativa Colombia aprende. Colombia. 


\section{ReiDoCrea. Volumen 1}

Moliner García, Odet. (2008). Condiciones, procesos y circunstancias que permiten avanzar hacia la inclusión educativa: retomando las aportaciones de la experiencia canadiense. REICE. Revista Iberoamericana sobre Calidad, Eficacia y Cambio en Educación, 27-44.

Organización de las Naciones Unidas para la Educación, la Ciencia y la Cultura. Declaración de Salamanca y Marco de Acción para las Necesidades Educativas Especiales. (1994).Salamanca, España. Ministerio de Educación y Ciencia.

Stainback, S. y Stainback, W. (1990). Inclusive schooling. En Stainback, S. y Stainback, W. (eds.): Support networks for inclusive schooling. Paul Brothers, 3-23. London.

Susinos Rada, Teresa (2002). Un recorrido por la inclusión educativa española. Investigaciones y experiencias más recientes. Revista de Educación no 327, pp. 49-68.

Vázquez Martínez, B., Méndez Pineda, J. y Mendoza Saucedo, F. (2010).Actitudes de Docentes Universitarios hacia los Procesos de Inclusión Educativa de Alumnos con Capacidades diferentes en la Universidad Autónoma de San Luís Potosí. En Primer Congreso Latinoamericano de Ciencias de la Educación. UABC México. 